Meta

Journal des traducteurs

Translators' Journal

\title{
Évaluation des définitions d'ouvrages
}

\section{Véronique Gesché}

Volume 42, numéro 2, juin 1997

Lexicologie et terminologie II (1) et Traduction et post-colonialisme en Inde

Translation and Postcolonialism: India (2)

URI : https://id.erudit.org/iderudit/002887ar

DOI : https://doi.org/10.7202/002887ar

Aller au sommaire du numéro

\section{Éditeur(s)}

Les Presses de l'Université de Montréal

ISSN

0026-0452 (imprimé)

1492-1421 (numérique)

Découvrir la revue

Citer cet article

Gesché, V. (1997). Évaluation des définitions d'ouvrages. Meta, 42(2), 374-390. https://doi.org/10.7202/002887ar
Résumé de l'article

Le présent article se penche sur le rôle de la définition et en dresse une typologie des types et des principaux défauts. On expose ensuite les résultats d'un volet de la recherche effectuée en 1993 par le CTB à la demande du ministère des Technologies nouvelles et de l'Emploi de la Région wallonne de Belgique. Enfin, on présente quelques recommandations à l'usage des auteurs de définitions. d'utilisation que vous pouvez consulter en ligne.

https://apropos.erudit.org/fr/usagers/politique-dutilisation/ 


\title{
ÉVALUATION DES DEFINITIONS D'OUVRAGES
}

VÉRONIQUE GESCHÉ

Centre de Terminologie de Bruxelles, Institut Libre Marie Haps, Bruxelles, Belgique

\begin{abstract}
Résumé
Le présent article se penche sur le rôle de la définition et en dresse une typologie des types et des principaux défauts. On expose ensuite les résultats d'un volet de la recherche effectuée en 1993 par le CTB à la demande du ministère des Technologies nouvelles et de l'Emploi de la Région wallonne de Belgique. Enfin, on présente quelques recommandations à l'usage des auteurs de définitions.
\end{abstract}

\begin{abstract}
In this article the author discusses the role of definitions, establishes a typology and indicates major shortcomings. She then presents results of one phase of the research carried out in 1993 by the BCT at the request of the Ministry of New Technologies and Employment in French-speaking Belgium. She concludes with helpful advice for writing definitions.
\end{abstract}

\section{LE RÔLE DES DÉFINITIONS DE MANUELS}

L'objectif premier d'un manuel technique ou scientifique est de transmettre des connaissances de manière plus ou moins vulgarisante et, partant, d'expliquer des concepts. Or l'examen de plusieurs ouvrages semble montrer qu'à la présence de définitions systématiques des termes nouveaux, l'auteur préfère souvent le recours au texte continu, aux paraphrases, etc., pour expliquer son sujet. Choix qu'il justifiera, à juste titre sans doute, par un souci de convivialité, l'éventuelle nécessité d'expliquer longuement les tenants et aboutissants d'un sujet, un désir d'éviter le style scolaire. Cependant, l'examen approfondi des termes expliqués et/ou définis (la frontière entre ces deux états étant précisément difficile à distinguer, nous le verrons) dans un échantillon d'ouvrages scientifiques et techniques montre que de nombreux manuels gagneraient sensiblement en clarté si la terminologie qu'ils manipulent et enseignent était un tant soit peu organisée. Sans doute ces constatations suffiraient-elles à susciter une réflexion générale sur la manière de concevoir un manuel, et elles sont une raison subsidiaire d'étudier les définitions. Rappelons que les rédacteurs des manuels d'utilisation, d'installation, de maintenance des équipements mais aussi les documentalistes d'entreprise et, les traducteurs techniques se recrutent très souvent parmi les spécialistes, moins frottés aux techniques de rédaction que les langagiers.

Second motif de cette analyse des définitions d'ouvrages spécialisés: les définitions sont le dernier recours des traducteurs confrontés à une notion inconnue, en langue étrangère ou dans leur langue maternelle, lorsque les dictionnaires sont incomplets ou erronés. Les définitions sont surtout la source d'inspiration des auteurs des dictionnaires scientifiques et techniques rédigeant leurs définitions et déterminant les degrés d'équivalence et d'homonymie des termes.

L'amélioration des activités terminographiques que sont les définitions des ouvrages spécialisés relève des tâches de l'édition scientifique et technique. Les terminographes devraient formuler un certain nombre de recommandations sur l'art de définir aux éditeurs spécialisés. 
Enfin, des logiciels automatisent depuis peu le repérage et le relevé des termes dans les ouvrages disponibles sur support informatique. On songe surtout au programme TERMINO de l'OLF. Le Centre de terminologie de Bruxelles a également écrit un petit programme, qui consulte les index d'ouvrages et recopie dans ces livres électroniques indexés les phrases entourant ou comportant les termes indexés. Ce programme rédige donc automatiquement des fiches de terminologie sur base des index élaborés par les auteurs des ouvrages. Pour dicter à ce programme ce qu'il doit copier dans le livre, une fois le pointeur positionné à chacune des pages dont le numéro figure dans l'index à côté des termes repris comme entrées, il fallait savoir si la majorité des définitions et des contextes utiles des livres spécialisés étaient rédigés en une, deux ou trois phrases. Nous devions savoir si le programme pouvait trouver lui-même une série de définitions sur la base d'indices typographiques: définitions en retrait, en italique, etc.

Les termes et leurs définitions (50 par ouvrage, soit 1250 en tout) ont été repérés et dépouillés à l'aide de l'index, s'il existait. En l'absence d'index, nous nous sommes basée sur les mises en évidence des termes et des définitions. Lorsque l'auteur n'avait pas mis ces définitions en exergue par une typographie spéciale, il a fallu dégager les termes par la structure interne des ouvrages (s'aider des titres, voire des paragraphes). Enfin, pour les textes presque «opaques», une lecture plus en profondeur s'est imposée.

\section{RAPPEL DE LA LITTÉRATURE SUR LES DÉFINITIONS}

Définir un terme par l'exemple, par comparaison ou par opposition avec d'autres contribuera peut-être à en faire apparaître plus clairement le sens. Cela ne constituera pas une base suffisante pour la formulation d'une définition lexicographique.

\section{Typologie des définitions} suivants :

Les définitions rencontrées dans toutes les monographies relèvent d'un des types

- Définition analytique : cette définition donne le super-ordonnant du terme à définir et indique ensuite les caractères ou sèmes définitoires qui situent le concept à définir, le défini, par rapport aux autres membres de cette classe. Exemple: PNEUMONIE: inflammation des tissus du poumon.

- Définition encyclopédique : elle comporte, outre les caractères définitoires, une série de sèmes décrivant tout ou partie des connaissances sur le (terme) défini.

- Définition par contenu : elle donne succinctement les parties du «tout» à définir. Cette définition peut se révéler suffisante dans certains cas, notamment pour les objets. Exemple: BICÉPHALE: qui a deux têtes.

- Définition par dénotation : elle est constituée d'une série exhaustive d'instances du concept. Exemple: TRAITEMENT DE DONNÉES : saisie, transformation et sortie de données.

- Définition opératoire : elle indique l'usage, la fonction, le mode d'emploi. Exemple : ORDINATEUR: machine destinée à des calculs rapides et en nombres très élevés.

- Définition paraphrastique : elle renvoie à une autre dénomination; elle est souvent utilisée pour les dérivés. Exemple : FLOTTAISON : action de faire flotter un objet.

- Définition descriptive : elle donne les caractères décrivant l'apparence extérieure du défini. Exemple: SINUS FRONTAUX: deux cavités pneumatiques asymétriques dans l'épaisseur de l'os frontal, séparées l'une de l'autre par une cloison.

- Définition par implication: elle utilise le mot dans un contexte explicatif. Exemple: DIAGNOSTIC: on établit un diagnostic lorsque l'on souhaite identifier certains symptômes en tant que caractéristiques de conditions spécifiques. 
- Définition par comparaison : elle souligne la similarité avec une notion connue. Exemple: COCHLÉE: sorte de spirale située dans l'oreille. Une de ses variantes est la définition par synonyme, donnant un synonyme plus connu du lecteur à la place du définissant. Exemple: DAISY : bellis perennis.

- Définition par contraste : elle définit par référence au contraire. Exemple: VIERGE : qui n'est pas formaté.

- Définition ostensive: elle montre par une image, un schéma, un graphique ou un exemple. Exemple : un ÉLECTROMYOGRAMME (voir ci-contre) montrera à quel point...

\section{Défauts fréquents des définitions}

Les règles rédactionnelles des définitions sont très importantes. Tous les usagers des dictionnaires les connaissent car elles ont été forgées au fil des siècles pour faciliter la lecture des dictionnaires. Des formulations définitoires lexicographiques pourraient également faciliter la compréhension des concepts ardus lors des lectures de monographies car elles sont aussi prévisibles que les indices visuels du lecteur, c'est-à-dire les signaux accélérant la lecture en permettant d'anticiper les mots à venir. En outre, de bonnes définitions systématiques simplifient grandement, voire éliminent, les développements sur les concepts scientifiques.

Pratiquement aucune des définitions d'ouvrages spécialisés n'échappe à une des critiques énoncées ci-dessous.

\section{Définition trop large}

Une définition de ce type peut s'appliquer à d'autres termes que celui qu'elle définit car elle ne mentionne pas assez de caractères qui distingueraient le concept de ses voisins. Exemple : SOURIS : dispositif permettant de déplacer le pointeur (les touches-flèches le peuvent également!).

\section{Définition trop restrictive}

Elle définit au contraire par des caractères trop précis ou par trop de caractères. Exemple: CROQUIS : représentation dessinée donnant une explication approximative d'un objet (un croquis n'est pas forcément accompagné d'un commentaire).

\section{Définition circulaire}

Cette définition recourt à un synonyme défini lui-même par le terme qu'il définit. Exemple: $\mathrm{H}_{2} \mathrm{O}:$ eau, qui consiste en deux atomes d'hydrogène et un atome d'oxygène (le générique est tautologique, il faudrait le remplacer par composé chimique).

\section{Définition par faux incluant}

Les définitions par genre prochain et différence spécifique peuvent comporter un incluant qui n'en est pas un. Voici les faux incluants relevés par J. Rey-Debove (1971) dans les dictionnaires de langue. Ils se retrouvent dans les ouvrages spécialisés et... dans une série de dictionnaires de spécialités, car la terminographie n'a pas repris tous les acquis de la lexicographie (ces défauts devraient notamment être présents à l'esprit des personnes recensant les dictionnaires spécialisés).

- Catégorie différente. Une telle définition ne définit pas un nom par un nom, un verbe par un verbe ou un groupe verbal, etc. Exemple: FORMATER : opération de préparation d'un disque pour lui permettre de recevoir des informations.

- Nombre différent. Ce type de définition comporte une autre erreur de syntaxe: un pluriel y est défini par un singulier, ou vice versa. Exemple: DÉVELOPPEMENT DE SYSTEME: toutes les activités humaines et mécaniques visant à créer de nouvelles applications. 
- Partie-incluant avec d'autres parties qualificatives. Ici, un tout est défini par une partie: même si la partie reprise comme «tout» est essentielle, elle ne lui est pas assimilable. Exemple: PATIN : semelle munie d'une lame métallique que l'on fixe sur la chaussure pour glisser sur la glace.

- Définition de la chose transformée. Dans cette définition génétique, le faux incluant représente le premier état (antérieur) de la chose transformée, et non sa catégorie englobante. Exemple: GLACE : eau congelée.

- Définition par la cause ou la conséquence. Exemple de confusion avec la cause : CONSTIPATrON : retard dans l'évacuation des selles. Exemple de confusion avec la conséquence: CONTRACEPTION : infécondité provoquée par les moyens anticonceptionnels.

- Faux incluant d'existence. Souvent, le générique adéquat (le super-ordonnant) est remplacé par une expression du type : absence de, manque de, défaut de... ou présence $d e . .$. comme s'il s'agissait là d'une classe logique à part entière. Or, la catégorie des «absences de» n'existe pas, dit J. Rey-Debove, et la plupart des définitions de ce type auraient pu exprimer l'inclusion. Exemple: GLYCOSURIE: trouble caractérisé par la présence de sucre dans l'urine, au lieu de présence de sucre dans l'urine. Le même phénomène $s$ "observe lors du recours à des expressions floues, malheureusement fort courantes : sorte de, espèce de, moyen de... (définitions par analogie). Exemples : CUED SPEECH: moyen de rendre plus complète... S'il est vrai que, pour des raisons linguistiques, le super-ordonnant d'un terme n'est pas exprimable dans tous les cas, l'observance de cette règle demeure capitale pour maintenir la clarté d'un domaine.

- Générique trop éloigné. Le terme est défini par un super-ordonnant qui est en réalité le super-ordonnant du défini ou celui du super-ordonnant de son super-ordonnant. Exemple: TABLE GRAPHIQUE: surface rectangulaire sur laquelle on pointe et dessine avec un photostyle. - Incluant inconnu. Le définissant n'a pas été défini, sans doute parce qu'on considère qu'il fait partie des prérequis. Exemple: RETOUR AUTOMATIQUE: retour qui... (à aucun autre endroit du livre le mot retour n'est défini).

- Absence d'incluant. Le terme à définir est englobé dans une phrase qui ne situera pas immédiatement le terme dans son système notionnel. Exemple : La DYSSYNTAXIE s'observe lorsque les structures syntaxiques sont mal utilisées; la PARAPHASIE MORPHOLOGIQUE consiste à remplacer un mot par un autre lui ressemblant par la forme. L'incluant se devine parfois aisément (pour le second exemple, paraphasie). Ce «défaut » est bien sûr fréquent dans les livres.

\section{Manque de systématicité}

Les définitions de tous les concepts d'une catégorie super-englobante doivent être cohérentes les unes par rapport aux autres (même nom de catégorie englobante pour les cohyponymes, etc.). Exemple: la définition d'une série d'appareils ne commence pas toujours par appareil...

\section{Portée non précisée}

Parfois, une définition ne s'applique qu'à un nombre limité de cas, n'est valable que pour une publication ou une école de pensée donnée. Cette limitation doit être mentionnée explicitement. Exemple: SAIGNEMENT: écoulement de sang ( $s^{\prime}$ utilise uniquement pour le nez, en médecine. Sinon, il faut utiliser «hémorragie»).

\section{Précision insuffisante}

Une précision accrue n'allonge pas forcément une définition; les ajouts de détails ne sont pas forcément nécessaires, ce sont souvent des termes scientifiques plus spécialisés qui le sont. Si la définition des termes construits avec-URIE mentionne par exemple la 
présence des composantes de l'urine par l'adjectif normal, l'usager du dictionnaire qui désire vérifier si les substances présentes dans l'urine en sont partie constituante ou si leur présence est accessoire ou pathologique (ASCORBURIE, COLIBACILLURIE, CYLINDRURIE, DIACÉTURIE, PEPSINURIE) sera induit en erreur par cette décision de l'auteur du dictionnaire.

\section{Concision insuffisante}

La définition d'un concept devrait être concise. Cette caractéristique étant souvent un corollaire inverse de la précédente, un compromis sera cherché entre concision et précision.

\section{Infraction à la syntaxe lexicographique}

L'ordre logique des renseignements fournis sur le concept doit être respecté :

- mention des traits définitoires dans la première phrase ou le premier syntagme, mention des traits accessoires éventuels dans une seconde phrase;

- citation des synonymes les plus courants avant les moins usités;

- en cas de polysémie, présentation du sens le plus général en premier lieu.

\section{Contextes}

En terminologie, on distingue trois types de contextes:

- contexte définitoire: définition non rédigée comme telle (définition par implication, notamment, ou définition encyclopédique);

- contexte explicatif : phrase(s) donnant des caractères secondaires du concept;

- contexte illustratif: montrant les collocations qu'il contribue fréquemment à former, son niveau de langue etc.;

- contexte-attestation : phrase servant uniquement à attester l'existence du défini.

Avec les contextes explicatifs, les contextes définitoires servent de base de rédaction pour les définitions terminographiques. Les contextes des ouvrages sont donc utiles aux programmes de dépouillement automatiques de corpora.

\section{OUVRAGES ANALYSÉS}

Notre choix s'est porté sur un corpus dont les définitions étaient susceptibles de répondre aux attentes des terminographes, à savoir:

- des ouvrages didactiques, destinés notamment à des étudiants de première année d'université, ainsi que des ouvrages publiés dans des collections de vulgarisation de haut niveau. On suppose en effet que ces ouvrages sont bien structurés;

- des ouvrages de collections faisant l'objet d'une politique d'édition précise, pour lesquels nos conclusions sont donc les plus susceptibles d'être utiles aux éditeurs;

- des livres de linguistique, car on supposait que cordonnier serait bien chaussé...;

- des ouvrages et manuels dont le feuilletage a permis de constater la présence de définitions.

Voici la liste des ouvrages étudiés :

BELISLE, C. et G. JOUANNADE (1988): Communication visuelle, Paris, Éditions d'Organisation.

collectif (1977): Troubles du langage: diagnostic et rééducation, Bruxelles, Pierre Mardaga.

COSTERMANS, J. (1980) : Psychologie du langage, Bruxelles, Pierre Mardaga.

D'HONDT, Th. et M. JADOUL (1988): Een eerste stap in de Programmeerwereld (Deel II), Schoonhoven, Academic service. 
DE BROCK, E. O. (1989): De Grondslagen van de Semantische Databases, Academic service.

DE GROOFF, D. (1991): Media Lexicon, Leuven, Lannoo - Mediacentrum.

DE GRUYTER (1989): Dictionaries - An International Encyclopedia of Lexicography, Berlin/New York, De Gruyter.

DE SCHAETZEN, C. (1990): Word 4 et 5 avancé. Les macro-commandes, coll. «Informatique», Alleur, Marabout.

DUMAS DE RAULY, Th. (1987): Choisir et utiliser les supports visuels et audiovisuels, Paris, Éditions d'Organisation.

FISHMAN, J. A. (1972): Advances in the Sociology of Language, Joshua A. Fishman.

GARDNER, H. (1980): Gribouillages et dessins d'enfants, Bruxelles, Pierre Mardaga.

GIBSON, E. J. et H. LEVIN (1976): Psychology of Reading, MIT Press.

HAMERS, J. F. et M. BLANC (1983): Bilingualité et bilinguisme, coll. «Psychologie et Sciences Humaines», Bruxelles, Pierre Mardaga.

IBM (1991): Manuel de Référence, DOS 5.0.

KORNELIS, I. J. et R. A. MOLLEMA (1989): Controle van de informatieverwerking, Brussel, Samson, Alphen-aan-den-Rijn.

LEROT, J. (1983) : Abrégé de linguistique générale, Louvain-la-Neuve, Cabay.

MOREAU, M.-L. et M. RICHELLE (1981): Acquisition du langage, Bruxelles, Pierre Mardaga.

MYKLEBUST, H. R. (1964): Psychology of Deafness, New York, London, Grune and Stratton.

OLDFIELD, R. C. et J. C. MARSHALL (1968) : Language, Modern Psychology, Harmondsworth, Penguin.

PAUL, B. (1988) : Efficiënter programmeren in Basic, MAKLU.

PIALOUX, P., VALTAT, M., FREYSS, G. et F. LEGENT (1975) : Précis d'orthophonie, Paris, Masson.

REY-DEBOVE, J. (1971): Étude linguistique et sémantique des dictionnaires français contemporains, Amsterdam, Mouton.

SHARPLES, M., HOGG, D., HUTCHISON, C., TORRANCE, S. et D. YOUNG (1989) : Computer and Thought, MIT Press.

VAN EIJCK, J. et E. THIJSEN (1989): Logica voor Alfa's en informatici, Academic service. ZGUSTA, L. (1971): Manual of Lexicography, The Hague/Paris, Mouton.

La petite taille de l'échantillon impose des réserves, même si le nombre de définitions analysées dans ce corpus est relativement élevé (plus de 1000 ).

\section{GRILLE D'ANALYSE}

Afin de cerner les caractéristiques des définitions, nous avons dressé deux grilles d'analyse. La première évaluait l'exploitabilité des définitions, telles qu'elles figurent dans les ouvrages, comme matériel terminographique. Elle listait les problèmes qualitatifs des définitions et explications de termes. La seconde, appliquée aux ouvrages pourvus d'un glossaire, était destinée à analyser la formulation des définitions (entre autres le pourcentage de définitions trop larges, restrictives, circulaires, ostensives, de définitions par comparaison, par catégorie lexicale différente, par l'exemple...). Mais deux grilles aussi précises étaient difficilement applicables. La première raison est la nécessaire liberté des auteurs pour la rédaction des définitions. La rareté des glossaires et des lexiques en fin d'ouvrage compromet également l'application d'une grille trop sévère: sur un rayon d'une grande librairie bruxelloise présentant 112 ouvrages d'un domaine, par exemple, seuls 7 livres comportaient un lexique ou un glossaire. En outre, le vocabulaire repris dans les 
glossaires ou lexiques porte souvent sur une partie de l'ouvrage, voire uniquement sur des prérequis à sa lecture.

Nous nous sommes donc bornée à évaluer les termes dans leur contexte définitoire, c'est pourquoi les termes rencontrés dans les ouvrages ont été soumis uniquement à la première grille. Celle-ci examinait la présentation formelle des termes et la manière dont l'auteur situait, le cas échéant, le terme dans son environnement conceptuel.

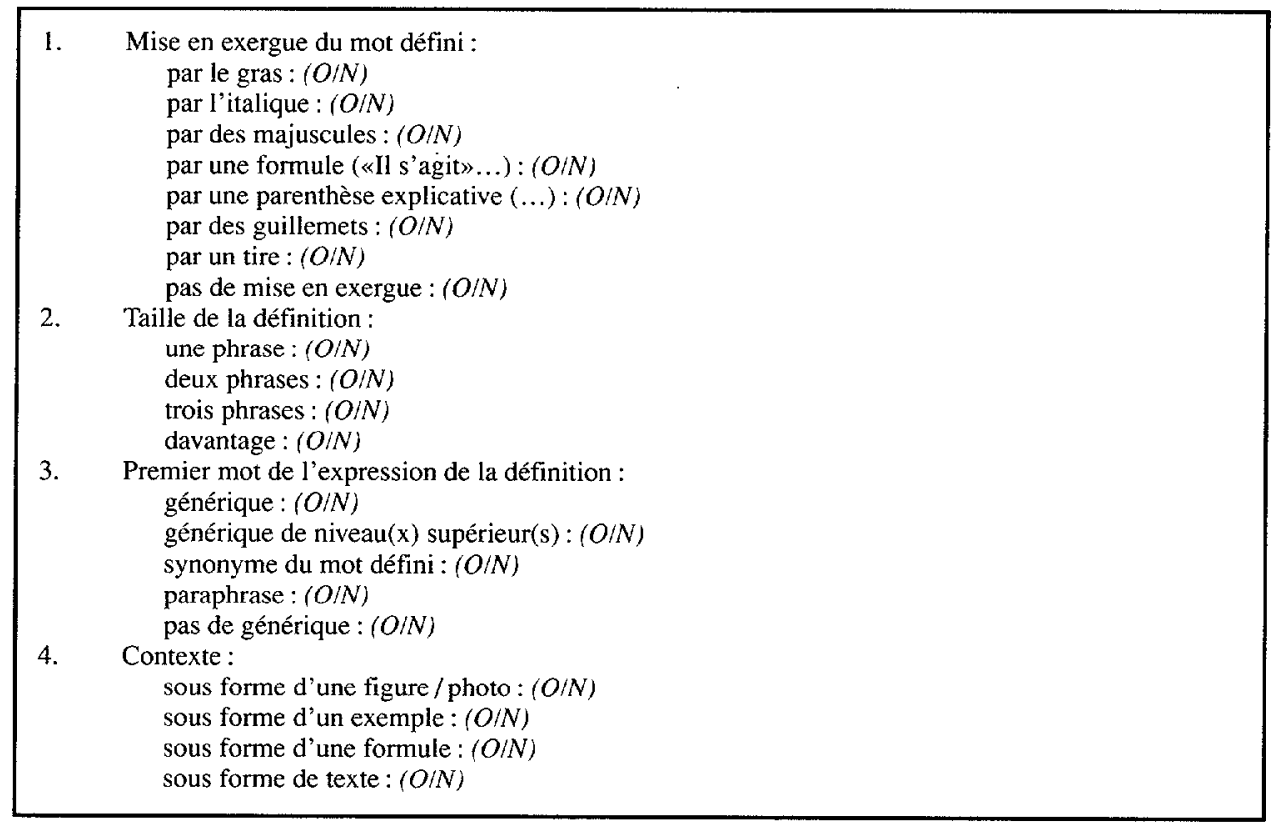

\section{ANALYSE DES DÉFINITIONS}

Où commence et où finit une définition? Les distances peuvent être telles que la définition se perd et que le terme est défini en dehors du paragraphe qui en traite:

- quelquefois, le terme à définir (qui figure dans un titre, par exemple) est séparé de sa définition par une digression. Celle-ci peut occuper jusqu'à plusieurs paragraphes;

- certaines définitions commencent avant l'apparition du terme: une énumération, par exemple, annonce par un générique les termes techniques à définir;

- dans une série énumérative de termes en exergue et définis, il se peut également qu'un terme, dans la foulée du raisonnement, ne soit pas défini, comme si l'auteur voulait briser la monotonie de cette série;

- enfin, il est impossible de dégager la définition d'un terme clé à la lecture de son con texte lorsque celui-ci est trop «dilué» ou trop pauvre.

Le repérage même des termes pose problème puisque les index comportent des notions techniques qui ne sont pas toujours définies et qu'à l'inverse, de très nombreux termes techniques définis dans le corps du livre ne figurent pas dans l'index. Problème accru lorsque l'index renseigne sur un terme dont le texte développe le thème mais qui ne 
figure même pas en tant que tel dans ce développement. Un autre problème de repérage est le «camouflage» de certains termes dans des formules discrètes comme : c'est le...; il consiste en...

\section{Mise en exergue des termes}

Si les termes sont mis en évidence aux pages du corps de l'ouvrage auxquelles ils sont définis, le programme pourra se borner à recopier la définition en même temps que le terme, en sautant tous les contextes d'occurrence du terme. Le second avantage, éditorial, de la typographie est d'attirer l'attention du lecteur sur les concepts nouveaux ou importants et sur leurs définitions. La mémorisation de ces termes par les apprenants en sera notamment facilitée.

Mise en exergue des termes par du gras

\begin{tabular}{|l|l|l|}
\hline RÉPONSE & NOMBRE & $\%$ \\
\hline oui & 282 & 23 \\
non & 968 & 77 \\
\hline TOTAL & 1250 & 100 \\
\hline
\end{tabular}

La mise en gras des termes est relativement fréquente : $23 \%$ des termes examinés.

Mise en exergue des termes par l'italique

\begin{tabular}{|l|l|l|}
\hline RÉPONSE & NOMBRE & $\%$ \\
\hline oui & $\mathbf{5 3 0}$ & 42 \\
non & 720 & 58 \\
\hline TOTAL & 1250 & $\mathbf{1 0 0}$ \\
\hline
\end{tabular}

Les termes sont souvent mis en évidence par l'italique (près de la moitié des termes examinés). Indépendamment de leur rôle, les caractères en italique sont plus fréquents dans l'édition que le gras parce qu'ils sont plus légers.

Mise en exergue des termes par des majuscules

\begin{tabular}{|l|l|c|}
\hline RÉPONSE & NOMBRE & $\%$ \\
\hline oui & $\mathbf{4 5}$ & $\mathbf{3}$ \\
non & 1205 & 97 \\
\hline TOTAL & $\mathbf{1 2 5 0}$ & $\mathbf{1 0 0}$ \\
\hline
\end{tabular}


Peu de termes ( $3 \%$ ) figurent en majuscules. Ce n'est pas étonnant : les capitales sont disgracieuses et cassent le rythme de la lecture.

Mise en exergue des termes par des guillemets

\begin{tabular}{|l|l|l|}
\hline RÉPONSE & NOMBRE & $\%$ \\
\hline oui & $\mathbf{5 8}$ & $\mathbf{5}$ \\
non & 1192 & 95 \\
\hline TOTAL & $\mathbf{1 2 5 0}$ & $\mathbf{1 0 0}$ \\
\hline
\end{tabular}

Les guillemets sont peu utilisés ( $5 \%$ ).

L'ensemble des termes mis en exergue par du gras, de l'italique, des majuscules et des guillemets s'élève donc à 984 , ce qui correspond à $79 \%$ des termes considérés.

Mise en exergue des termes par un titre

\begin{tabular}{|l|l|l|}
\hline RÉPONSE & NOMBRE & $\%$ \\
\hline oui & 69 & 6 \\
non & 1181 & 94 \\
\hline TOTAL & 1250 & 100 \\
\hline
\end{tabular}

Les titres annoncent $6 \%$ des termes seulement.

Mise en exergue des termes par une formule

\begin{tabular}{|l|l|l|}
\hline RÉPONSE & NOMBRE & $\%$ \\
\hline oui & 198 & 16 \\
non & 1052 & 84 \\
\hline TOTAL & 1250 & 100 \\
\hline
\end{tabular}

Un autre procédé de mise en évidence des termes est sémantique: il s'agit de formules définitoires, dont l'éventail est déjà nettement plus varié. Il faudra donc que les programmes de dépouillement automatique prennent en compte un maximum de phrases du type: $I l$ s'agit de; On appelle $X \ldots$.. Ce mode d'introduction n'est pas fréquent ( $16 \%)$, ce qui est d'ailleurs préférable pour notre propos: il est impossible de trouver automatiquement des formules variables. 
Mise en exergue des termes par une parenthèse explicative

\begin{tabular}{|l|l|l|}
\hline RÉPONSE & NOMBRE & $\%$ \\
\hline oui & $\mathbf{6 3}$ & 5 \\
non & 1187 & 95 \\
\hline TOTAL & 1250 & 100 \\
\hline
\end{tabular}

La parenthèse explicative n'est pas un repère possible des définitions, vu les «déchets» que constituent les parenthèses non explicatives. Elle est rare $(5 \%)$.

Pas de mise en exergue des définitions

\begin{tabular}{|l|l|l|}
\hline RÉPONSE & NOMBRE & \% \\
\hline oui & $\mathbf{3 5}$ & $\mathbf{3}$ \\
non & 1215 & 97 \\
\hline TOTAL & $\mathbf{1 2 5 0}$ & $\mathbf{1 0 0}$ \\
\hline
\end{tabular}

On constatera le nombre relativement élevé de termes définis par une formule plus ou moins claire : 296 , soit $24 \%$ des termes. Pour trouver ces définitions, moins accessibles, l'index est donc indispensable.

\section{Taille}

Afin qu'un programme puisse recopier automatiquement un nombre standard de phrases pour la définition, la taille la plus fréquente des définitions doit être connue. Nous pouvons la comparer aux présupposés théoriques dont nous disposons en la matière, à savoir qu'une bonne définition est généralement assez courte, qu'elle comporte idéalement une ou deux phrases, dont la seconde présente les caractères accessoires.

Définitions d'une seule phrase

\begin{tabular}{|l|l|l|}
\hline RÉPONSE & NOMBRE & $\%$ \\
\hline oui & $\mathbf{8 0 1}$ & $\mathbf{6 4}$ \\
non & 449 & 36 \\
\hline TOTAL & $\mathbf{1 2 5 0}$ & $\mathbf{1 0 0}$ \\
\hline
\end{tabular}

La taille de la définition est généralement assez réduite : une phrase dans $64 \%$ des définitions examinées. 
Définitions de deux phrases

\begin{tabular}{|l|l|l|}
\hline RÉPONSE & NOMBRE & $\%$ \\
\hline oui & 210 & 17 \\
non & 1040 & 83 \\
\hline TOTAL & 1250 & 100 \\
\hline
\end{tabular}

Les définitions en deux phrases sont plus rares : $17 \%$.

Définitions en trois phrases

\begin{tabular}{|l|l|l|}
\hline RÉPONSE & NOMBRE & $\%$ \\
\hline oui & $\mathbf{6 5}$ & $\mathbf{5}$ \\
non & 1185 & 95 \\
\hline TOTAL & $\mathbf{1 2 5 0}$ & $\mathbf{1 0 0}$ \\
\hline
\end{tabular}

Les définitions en trois phrases sont très rares : $5 \%$.

Le nombre de définitions composées de deux ou trois phrases est de 275 , soit $22 \%$ des définitions.

Définitions de plus de trois phrases

\begin{tabular}{|l|l|l|}
\hline RÉPONSE & NOMBRE & $\%$ \\
\hline oui & $\mathbf{1 7 4}$ & $\mathbf{1 4}$ \\
non & 1076 & 86 \\
\hline TOTAL & $\mathbf{1 2 5 0}$ & $\mathbf{1 0 0}$ \\
\hline
\end{tabular}

Les définitions de plus de trois phrases sont plus nombreuses que celles de trois phrases: $14 \%$.

Ce critère de taille est parfois difficile à évaluer: on hésite à considérer certains développements qui suivent une définition comme faisant partie intégrante de celle-ci. Ces chiffres inclinent donc à prendre deux phrases comme unité de sélection standard pour les programmes de lecture d'index.

\section{Premier mot}

Ce critère donne une idée de la proportion de définitions directement utilisables, c'est-à-dire commençant par un générique, même si le niveau de ce générique est éloigné. Il révèle également les définitions qui nécessiteront une reformulation complète par le 
terminographe pour pouvoir figurer dans son dictionnaire : premier mot constitué d'un synonyme du terme défini, l'absence de générique ou encore expression paraphrasée (explication).

Définitions dont le premier mot est le générique du défini

\begin{tabular}{|l|l|l|}
\hline RÉPONSE & NOMBRE & $\%$ \\
\hline oui & $\mathbf{6 2 9}$ & 50 \\
non & 621 & 50 \\
\hline TOTAL & $\mathbf{1 2 5 0}$ & $\mathbf{1 0 0}$ \\
\hline
\end{tabular}

La proportion de définitions analytiques est de $50 \%$. La proportion importante de ces dléfinitions, qui témoignent d'un souci de placer le terme dans une hiérarchie de concepts, est plutôt rassurante.

Définitions dont le premier mot est un générique de niveau(x) supérieur(s)

\begin{tabular}{|l|l|l|}
\hline RÉPONSE & NOMBRE & $\%$ \\
\hline oui & 213 & 17 \\
non & 1037 & 83 \\
\hline TOTAL & 1250 & 100 \\
\hline
\end{tabular}

Certaines définitions, toutefois, commencent par un générique de niveau trop élevé, ce qui est un peu trop souvent le cas, si l'on en croit les résultats $(17 \%)$.

Définitions dont le premier mot est un synonyme du défini

\begin{tabular}{|l|l|l|}
\hline RÉPONSE & NOMBRE & $\%$ \\
\hline oui & 122 & 10 \\
non & 1128 & 90 \\
\hline TOTAL & 1250 & 100 \\
\hline
\end{tabular}

Nous serons d'autre part heureusement frappée par l'usage peu fréquent (10\%) de synonymes, auxquels recourent les auteurs qui ne veulent pas alourdir leur texte: des définitions seront parfois constituées exclusivement d'un synonyme. 
Définitions dont le premier mot est une paraphrase (texte) du défini

\begin{tabular}{|l|l|l|}
\hline RÉPONSE & NOMBRE & $\%$ \\
\hline oui & $\mathbf{2 3 2}$ & $\mathbf{1 9}$ \\
non & 1018 & 81 \\
\hline TOTAL & $\mathbf{1 2 5 0}$ & $\mathbf{1 0 0}$ \\
\hline
\end{tabular}

Quant aux autres définitions, elles sont souvent formulées de manière très libre (19 \% de paraphrases) et comportent dans ce cas plus de trois phrases. Ce cas de figure, taille élevée et paraphrase, est le type de définition à la fois le plus confus et le moins exploitable.

D'autre part (et cette remarque est capitale), plus la définition est courte, plus on constate la présence du bon générique en son début. Tandis qu'une définition plus étendue sera, comme nous venons de le constater, caractérisée par l'usage d'une paraphrase. Cela confirme que l'auteur s'étend davantage s’il ne recourt pas à la meilleure technique de rédaction pour ses définitions.

Définitions dont le premier mot n'est pas un générique du défini

\begin{tabular}{|l|l|l|}
\hline RÉPONSE & NOMBRE & $\%$ \\
\hline oui & $\mathbf{5 4}$ & $\mathbf{4}$ \\
non & 1196 & 96 \\
\hline TOTAL & $\mathbf{1 2 5 0}$ & $\mathbf{1 0 0}$ \\
\hline
\end{tabular}

Enfin, la catégorie réduite ( $4 \%$ ) de définitions sans générique reprend les définitions dont le premier mot ou la première expression significative (idéalement, le générique) est absent, comme s'il était sous-entendu : l'ADJuvant indique que...

\section{Contexte}

L'environnement du terme et de sa définition est incontournable, étant donné la difficulté de repérer automatiquement les définitions. Ce contexte constitue par ailleurs la preuve d'utilisation de la dénomination. Enfin, il est intégré avec ou sans définition dans la plupart des banques de terminologie dès qu'il est significatif.

Le contexte est encore plus complexe à délimiter que la définition elle-même. Certains termes apparaissent en outre sans générique ni contexte.

Contextes consistant en un exemple

\begin{tabular}{|l|l|l|}
\hline RÉPONSE & NOMBRE & $\%$ \\
\hline oui & $\mathbf{3 0 3}$ & $\mathbf{2 4}$ \\
non & 947 & 76 \\
\hline TOTAL & 1250 & 100 \\
\hline
\end{tabular}


Le contexte significatif pour les dictionnaires consistera le plus souvent en un exemple, qui complète et clarifie la définition. Ce type est fréquent dans notre échantillon: $24 \%$ des termes étudiés.

Contextes consistant en une figure ou une photo

\begin{tabular}{|l|l|l|}
\hline RÉPONSE & NOMBRE & $\%$ \\
\hline oui & 191 & 15 \\
non & 1059 & 85 \\
\hline TOTAL & 1250 & 100 \\
\hline
\end{tabular}

Les figures et les photos ( $15 \%$ de notre échantillon) constituent bien sûr un type de contexte inexploitable et impossible à détecter par un programme. Ce programme les «sautera» au profit des phrases qui les suivent immédiatement (phrases qui ne seront donc pas significatives).

Contextes consistant en une formule

\begin{tabular}{|l|l|l|}
\hline RÉPONSE & NOMBRE & $\%$ \\
\hline oui & 14 & 1 \\
non & 1236 & 99 \\
\hline TOTAL & 1250 & 100 \\
\hline
\end{tabular}

Les formules mathématiques, heureusement peu nombreuses, ne constituent pas des contextes significatifs pour un dictionnaire sur papier ou électronique.

Contextes consistant en un développement encyclopédique

\begin{tabular}{|l|l|l|}
\hline RÉPONSE & NOMBRE & $\%$ \\
\hline oui & 166 & 13 \\
non & 1084 & 87 \\
\hline TOTAL & 1250 & 100 \\
\hline
\end{tabular}

La catégorie «forme textuelle» indique les $13 \%$ de termes dont la définition proprement dite est suivie d'un développement. Ce type de contexte, d'une longueur parfois très grande, ne gagnerait pas à être copié. Le programme le coupe de toutes manières puisqu'un nombre fixe de phrases lui est prescrit pour ses copies. 


\section{RECOMMANDATIONS AUX AUTEURS}

Pour les contributions originales à la science et à la technique, il n'est pas question d'emprisonner les éditeurs et, par eux, les auteurs, dans des contraintes rédactionnelles. D'autant moins que définitions et contextes ne sont repris que comme «pièces» du dossier du terme, avant, pour la définition, d'être remaniée à l'autre bout de la chaîne terminographique, et ce, pour des raisons de règles internes de confection des dictionnaires, de banques de données, et pour respecter les droits d'auteurs. Il ne s'agit donc pas de citer dans les dictionnaires des définitions de manuels mais de disposer de matériaux fiables quant au contenu.

Via les éditeurs, directeurs de collection et secrétaires de rédaction, les conseils s'adresseraient plutôt aux auteurs publiant dans des collections de vulgarisation, y compris des manuels utilisés dans les cours de première année de l'enseignement universitaire. Ces auteurs sont en effet déjà soumis à des contraintes éditoriales incarnant la philosophie de la collection. Les recommandations formulées ci-après amélioreraient et systématiseraient la rédaction des ouvrages sans l'entraver ou la rendre scolaire.

\section{Mise en exergue du terme}

Certains ouvrages font l'effort de distinguer les termes techniques, comme dans ce manuel de sémantique lexicale, en les faisant précéder d'un signe cabalistique lorsqu'ils apparaissent pour la première fois et qu'ils ne sont guère usités en dehors de l'ouvrage.

Certains manuels sont dépourvus d'index et ne mettent pas en exergue les termes techniques. Ainsi, l'un des manuels examinés a défini de nombreux termes techniques, mais sans les distinguer par une mise en gras ou en italique, alors que d'autres termes importants du même ouvrage figuraient en italique parce qu'ils étaient importants, mais ils n'ont pas été définis. Il y a donc ici un potentiel bien présent mais inexploitable par les terminographes. D'autres manuels définissent, parmi une masse de mots en italique, l'un d'entre eux, sans que cette définition soit directement accessible au lecteur désireux de se rafraîchir la mémoire, par exemple. Sans parler des manuels qui mettent en évidence les termes techniques (les organes de l'audition dans Que sais-je?, par exemple) mais se contentent de les citer comme faisant partie de telle ou telle figure, sans que l'on puisse en déduire leur fonction ou signification.

Seconde constatation: un index permet à l'auteur de vérifier la systématicité des notions exposées et la cohérence de sa terminologie.

Ensuite, la première et meilleure façon de permettre un repérage aisé des termes consiste à les mettre en forme (la mise en gras facilite le repérage visuel, l'italique ayant déjà de nombreuses fonctions). La mise en évidence typographique, dans le corps de l'ouvrage, de l'occurrence définie présente un double intérêt : repérage par le lecteur et par le programme, mise en exergue plus claire que les procédés de désignation courants (mise en gras, dans l'index, du numéro de page de l'occurrence où le terme est défini).

\section{Présence de la définition}

On peut regretter que les termes très spécifiques ne soient pas tous définis.

Dans certains ouvrages scientifiques, l'auteur intitule explicitement certains paragraphes «Définition», afin de permettre un repérage plus rapide et efficace des termes techniques; en réalité, le contenu du paragraphe ressemble davantage à une explication vulgarisante qu'à une réelle définition du terme.

\section{Taille de la définition}

Les termes techniques devraient être expliqués en moins d'un paragraphe (trois ou quatre phrases tout au plus). Cela constitue également un important critère de clarté dans un ouvrage de vulgarisation. 


\section{Formulation de la définition}

À côté de la taille, la forme de la définition constitue un critère délicat: il faudrait éviter le plus possible que l'un des éléments de la définition, en l'occurrence le générique qui doit "présider", ne se trouve beaucoup plus haut ou plus bas dans le texte (ce qui s'observe dans des effets de style ou pour les besoins d'une énumération). À éviter aussi, si possible: l'intercalation d'éléments secondaires entre deux phrases de la définition. Si ces deux derniers conseils semblent incompatibles avec le style et la démarche mentale de certains auteurs, ils ne sont pas motivés uniquement par le nombre de phrases fixes que doit copier notre programme mais aussi, du point de vue didactique, par les imprécisions et longueurs dans l'expression qu'ils visent à combattre.

Les manuels pourvus d'un glossaire ou de termes définis de manière assez systématique ont également été examinés d'un point de vue terminologique. Ces ouvrages offrent une clarté plus grande, mais les règles de rédaction des définitions n'ont pas non plus été observées dans tous les glossaires. Selon les ouvrages, les définitions des glossaires se révèlent soit trop larges soit trop restrictives; plusieurs définitions sont circulaires ou trop vagues. Parmi ces cas de figure, de nombreuses combinaisons sont possibles: une définition peut à la fois être trop large et recourir à un faux incluant d'existence; être à la fois trop restrictive et agrammaticale, etc. De même, l'auteur devrait définir correctement et de manière complète les termes techniques que comporte l'index: le lecteur (ou le terminographe) qui a rencontré dans l'index des termes dont traite l'ouvrage mais qu'il comprend rnal préférerait bien sûr une définition à un synonyme approximatif ou quelques exemples. Sur base des règles de rédaction terminologiques, rares sont donc les définitions parfaites mais un livre n'est pas un dictionnaire...

Si l'auteur ne désire pas couper le rythme de la lecture par des définitions lexicographiques, quantité de formules permettent d'insérer des définitions claires et concises dans un texte. En atteste le nombre d'ouvrages utilisant des expressions telles que : par $X$, l'auteur désigne $Y . .$. ; par $X$, on entend $Y . .$. ; on appelle $X$ l' $Y . .$. ; on parlera de $X$ lorsque...; il s'agit d' un... Sans parler des possibilités offertes par la ponctuation, les listes numérotées (traits principaux d'une maladie, par exemple) ou la syntaxe (définir un terme dans une proposition incise). Exemples: la zone de Broca, contrôlant l'émission du langage articulé... ; commissurotomie (c'est-à-dire section des commissures).

Dans l'hypothèse idéale d'une collaboration étroite entre éditeurs et terminographes où ces derniers disposeraient dès avant la publication du manuscrit sur traitement de texte, l'auteur pourrait en outre mettre ses définitions en texte masqué (texte qui peut être affiché sans être imprimé, en traitement de texte), pour permettre sa copie.

L'inclusion de définitions valables d'un point de vue terminologique ne constitue bien entendu pas la préoccupation principale des auteurs et des éditeurs; en outre, la facilité d'élaboration des définitions au sein d'un ouvrage scientifique dépendra à la fois du domaine concerné (à la différence de l'informaticien rédigeant un manuel de traitement de texte, le spécialiste en psychopathologie se verra sans doute contraint à davantage de littérature pour circonscrire ses concepts), de la technicité de la matière abordée et de la collection ou de l'auteur. Les auteurs de manuels à caractère scientifique et technique très didactiques, les auteurs de notes de cours publiées amélioreraient la qualité de leurs définitions récolteraient le fruit de leurs efforts: reconnaissance des lecteurs, d'une part, exploitation subséquente de leur terminologie escortée de droits d'auteurs dérivés, d'autre part.

Quelle serait pour la terminographie la définition idéale? Elle se composerait d'un maximum de trois phrases et serait repérable par une typographie particulière; elle commencerait par le générique immédiat (lorsque c'est linguistiquement possible); elle ne serait ni trop générale ni trop précise. Autant que faire se peut, elle serait acceptable sur les plans lexicographique et syntaxique. Le contexte serait laissé à l'appréciation du 
terminographe, qui évaluerait la longueur de celui qu'il prélèverait en fonction des besoins de la banque de données terminologiques réceptrice.

Et les contraintes éditoriales? La conformité à notre «microlangage» n'empêche pas les éditeurs d'édicter leurs propres instructions; au contraire, elle assurerait une uniformité stylistique, que les auteurs ne tarderaient pas à trouver pratique et qui ne déplairait pas aux lecteurs désirant obtenir des informations claires; les éditeurs verraient leur travail de relecture et/ou de réécriture facilité puisque, au sein d'une même collection, les bases en seraient plus proches d'un auteur à l'autre.

\section{EXPLOITATION DES DÉFINITIONS PAR LE PROGRAMME DE LECTURE D'INDEX}

En attendant cette harmonisation et comme nous nous y attendions, la recopie des définitions et des contextes donnera donc une moisson variable, y compris au sein d'un même ouvrage, du moins si des comptages ultérieurs confirment les chiffres de notre échantillon:

- peu de livres comportent un glossaire, lequel n'est en outre pas complet. Il n'est donc paradoxalement pas prudent d'exploiter cette source;

- dans le corps de l'ouvrage, la définition du terme est souvent inexistante, incomplète parce que entrelardée d'une ou deux phrases digressives, ou alors elle ne peut être trouvée parce que le défini n'est pas repris dans l'index;

- les définitions ne seront pas toutes canoniques, tant s'en faut;

- l'environnement du terme n'est pas toujours exploitable (photos, formules, dessins); il peut être inexistant, pauvre ou «dilué»;

- parmi les termes définis, $79 \%$ font l'objet d'une mise en évidence typographique (gras, italique, majuscules ou guillemets). Ils peuvent donc être repérés même s'ils ne figurent pas dans l'index, à moins que l'écriture d'attention choisie dans l'ouvrage ne soit également utilisée à d'autres fins que la seule valorisation des termes (italique). Les termes mis en évidence par un titre, une parenthèse explicative ou une formule ne peuvent par contre être repérés automatiquement que grâce à l'index ;

- nous pouvons prescrire à notre programme de copier deux phrases pour les définitions qu'il a repérées; comme la plupart de ces définitions se trouveront dans la première phrase, elles seront donc effectivement épinglées. Pour plus de sécurité, nous faisons également copier par le programme les deux phrases précédant l'occurrence;

- parmi ces définitions, $50 \%$ seront bien formées (par genre effectivement prochain et différence spécifique);

- en l'absence de définitions, $25 \%$ des contextes pourront être repris tels quels (exemples et formules) dans les banques de terminologie. Leur consignation automatique accélérera également la rédaction des définitions.

Les matériaux récoltés par notre programme de lecture d'index ne sont pas utilisés directement, rappelons-le. Ils ne sont que de la documentation destinée au travail, prépondérant, de synthèse et d'abstraction de l'auteur du dictionnaire. Le terminographe vérifiera et complétera l'index. Il rerédigera les définitions les plus complètes et les plus conformes aux canons terminographiques, afin de faire de sa terminologie un ensemble cohérent. Mais ce qui rend notre programme de lecture d'index et les concordanciersdépouilleurs de textes comme TERMINO efficaces comme réservoirs documentaires, c'est leur rapidité : leurs récoltes peuvent être complétées en tout temps par les définitions et les contextes d'autant d'ouvrages indexés que le souhaite l'auteur de dictionnaires.

\section{RÉFÉRENCE}

REY-DEBOVE, J. (1971) : Étude linguistique et sémiotique des dictionnaires français contemporains, La Haye, Mouton, $330 \mathrm{p}$. 